\section{Information for Authors}

The Canadian Journal of Neurological Sciences publishes original articles in the clinical and basic neurosciences. Manuscripts are considered for publication with the understanding that, except for identified review articles, they have not been published elsewhere except in abstract form and are not under simultaneous consideration by another publication. Manuscripts should be submitted to:

The Editor

Canadian Journal of Neurological Sciences

P.O. Box 4220

Station C

\section{Calgary, Alberta, Canada T2T 5N1}

Manuscripts and all illustrations should be submtted in triplicate. Papers will be accepted in English or French. All papers should be accompanied by an abstract or a résumé of approximately 150 words on a separate page, preferably in both languages, although the Journal will provide the translation if requested. All manuscripts should be double spaced throughout, including references and legends for illustrations. Margins of at least $25 \mathrm{~mm}$ should be left on all sides.

For detailed instructions regarding style and layout, authors should refer to "Uniform requirements for manuscripts submitted to biomedical journals". Copies of this document may be obtained by writing to the Journal office, but the main points will be summarized here. Articles should be subdivided under conventional headings of "introduction", "methods and materials", "results" and "discussion" but other headings and subheadings will be considered if more suitable for a particular manuscript. A title page should identify the title of the article, authors, name of institution(s) from which the work originated, and the address and telephone number of the author to whom communications should be addressed. Pages of text should be numbered consecutively. Acknowledgements, including recognition of financial support, should be typed on a separate page at the end of the text.

References are to be numbered in the order of citation in the text. Those cited only in tables or in legends for illustrations are numbered in accordance with a sequence established by the first identification in the text of a particular table or illustration. Titles of journals should be abbreviated according to the style used in Index Medicus. References should be complete including the names of the first three authors followed by "et al" if there are more than three authors, full title, year of publication, volume number, and inclusive pagination for journal articles. Book or chapter references should also include the place of publication and name of the publisher. Examples of correct forms of references follow:

\section{Journals}

Poirier LJ, Filion M, Larochelle L, et al. Physipathology of experimental parkinsonism in the monkey. Can J Neurol Sci 1975; 2: 255-263.

\section{Chapter in a book}

McGeer PL, MCGeer EG. Amino acid neurotransmitters. In: Siegel Gl, Albers RW, Agranoff BW, Katzman R, eds. Basic Neurochemistry. Boston: Little, Brown \& Co, 1981: 233-254.

llustrations should be high quality glossy black-and-white photographic prints, preferably $127 \times 173 \mathrm{~mm}\left(5 \times 7^{\prime \prime}\right)$. Original artwork and radiographs should not be submitted. The additional cost of colour illustration must be borne by the author; quotations are available upon request from the Journal office. All figures should be identified on the back with the author's name and figure number. Letters and arrows applied to the figures to identify particular findings should be professional appliques suitable for publication. Photomicrographs should include a calibration bar with the scale indicated on the figure or in the legend. Legends for illustrations should be typed on a separate page from the illustrations themselves.

Tables should each be on a separate page and be identified with the title or heading. Particular care should be taken in the preparation of tables to ensure that the data are presented in the most clear and precise format. Each column should have a short or abbreviated heading. Place explanatory matter in footnotes, not in the heading. Do not submit tables as photographs.

The SI system (système international d'unités) should be used in reporting all laboratory data, even if originally reported in another system. Temperatures are reported in degrees Celsius. Other measurements should be reported in the metric system. English language text may use either British or American spelling, but should be consistent throughout.

Review articles on selected topics also are published by the Journal. These are usually invited, but unsolicited reviews will be considered. It is suggested that authors intending to submit reviews contact the Editor in advance.

Letters to the Editor are welcome. These should be limited to two double-spaced pages and may include one illustration and a maximum of four references.

\section{Information aux Auteurs}

Le journal Canadien des Sciences Neurologiques publie des articles originaux dans les sciences neurologiques, cliniques et fondamentales. Les manuscrits ne sont considérés pour publication qu'à la condition expresse, à l'exception des articles de revue clairement identifies comme tel, qu'ils n'aient pas été publiés ailleurs, sauf sous forme de résumé et qu'ils ne soient pas sous consideration simultanée par un autre journal. Les manuscrits doivent être soumis à:

L'Editeur

Journal Canadien des Sciences Neurologiques,

P.O. Box 4220,

Station C,

\section{Calgary, Alberta, Canada T2T 5N1}

Les manuscrits et toutes les illustrations doivent être soumis en triplicata. Les articles seront acceptés en français ou en anglais. Tous les articles doivent être accompangés d'un résumé d'environ 150 mots, sur page séparée, préférablement dans les deux langues, quoique le journal puisse fournir cette traduction sur requête. Les manuscrits doivent être dactylographiés complètement à double interligne y compris les références et les légendes pour illustrations. Des marges d'au moins $25 \mathrm{~mm}$ doivent être laissées de tous les côtés.

Pour les conseils plus détaillés sur le style et la présentation du texte, les auteurs doivent se référer au texte intitulé "Règlements uniformes pour les manuscrits soumis aux journaux biomédicaux". On peut obtenir une copie de ce document en écrivant au bureau du Journal, mais en voici les principaux points: Les articles doivent être présentés selon le plan habituel: "Introduction", "Matériel et méthodes", "Résultats" et "Discussion", mais il est possible d'employer d'autres titres ou sous-titres si nécessaire pour un manuscrit en particulier. Sur une page titre séparée on doit identifier le titre de l'article, les auteurs, les institutions d'où origine le travail, ainsi que l'adresse et le numéro de téléphone de l'auteur à qui devront être adressées les communications. Les remerciements, incluant ceux pour l'appui financier, doivent être dactylographiés sur page séparée à la fin du texte. Les références doivent être numérotées dans l'ordre où elles sont citées dans le texte. Celles qui sont citées seulement dans les tableaux ou légendes d'illustrations sont numérotées selon la séquence établie par la première identification dans le texte de ces tableaux ou illustrations particulières. Les titres des Journaux doivent être abrégés selon le style utilisé dans Index Medicus. Les références doivent être complètes, incluant le nom des trois premiers auteurs suivis de "et al", s'il y a plus de trois auteurs, le titre complet, l'année de publication, le numéro du volume et les premières et dernières pages de l'article. Les références aux livres et chapitres de livres doivent aussi inclure le lieu de la publication et le nom de la maison d'édition. Les exemples corrects suivants peuvent être utilisés:

\section{Journaux}

Poirier LJ, Filion M, Larochelle L, et al. Physiopathology of experimental parkinsonism in the monkey. Can I Neurol Sci 1975; 2: 255-263.

\section{Chapitre de livre}

MCGeer PL, MCGeer EG, Amino acid neurotransmitters. In: Siegel Gl, Albers RW, Agranoff BW, Katzman R, eds. Basic Neurochemistry. Boston: Little, Brown \& Co, 1981: 233-254.

Les illustrations doivent être sur papier brillant de haute qualité et imprimés en blanc et noir, préférablement $127 \times 173 \mathrm{~mm}\left(5 \times 7^{\prime \prime}\right)$. Les illustrations et photographies originales ne doivent pas être soumises. Le coût supplémentaire des illustrations en couleur revient entièrement à l'auteur: les coûts détaillés peuvent être obtenus directement au bureau du Journal. Il faut identifier toutes illustrations en inscrivant au dos le nom de l'auteur et le numéro. Toutes lettres ou flèches appliquées aux illustrations pour identifier un aspect particulier doivent être de qualité professionnelle. Les photomicrographies doivent inclure une barre de calibration dont l'échelle est mentionée dans la légende. Les légendes des illustrations doivent être dactylographiées sur une page séparée de celles-ci.

Les tableaux doivent être sur des pages séparées et être identifiés avec titre. On doit prendre un soin particulier dans la préparation de ces tableaux afin d'assurer que les données soient présentées avec le format le plus clair et le plus précis possible. Chaque colonne doit avoir un court titre. Les explications doivent être placées en dessous du tableau et non en sous-titre. Un tableau ne doit pas être soumis sous forme de photographie.

On doit employer le système international d'unités (SI) pour toutes données de laboratoire, même si celles-ci sont originellement présentées dans un autre système. Les températures doivent être citées en degrés Celcius. Les autres données doivent utiliser le système métrique. Les textes en anglais peuvent utiliser l'orthographe anglais ou américain, mais cet usage doit être constant.

Le Journal publie également des articles de revue sur des sujets sélectionnées. Ces articles sont généralement sur invitation, mais, à l'occasion, une revue non sollicitée peut être acceptée. Il serait préférable que les auteurs ayant l'intention de soumettre une telle revue contactent d'abord l'Editeur.

Nous accueillons les lettres à l'Editeur. Celles-ci doivent se limiter à deux pages, double interligne et peuvent contenir une seule illustration et ne citer q'un maximum de quatre références. 